\title{
A Novel Method for Segmentation of QRS Complex on ECG Signals and Classification of Cardiovascular Diseases via a Hybrid Model Based on Machine Learning
}

\author{
Eftal Sehirli $^{* 1}$, Muhammed Kamil Turan ${ }^{2}$
}

\begin{abstract}
Submitted: 14/11/2020 Accepted : 08/02/2021
Abstract: Automated-detecting intelligent programs and methods are developing to find out diseases in medicine in recent years. Developing new methods and improving existing ones are currently ongoing research. One of the most important health problems is heart diseases for all people in the world. Electrocardiography (ECG) is a diagnosis tool that gives substantially functional information about heart and cardiac system. In this work, it is primarily aimed at developing an intelligent system based on ECG signal processing, analysis, and classification via a hybrid machine learning model. This work uses 837 ECG signal fragments that includes 7 different classes shared in MIT-BIH Arrhythmia database for one lead. The ECG signals are applied on a preprocessing to smooth signals and correct baselines. $\mathrm{Q}, \mathrm{R}$ and $\mathrm{S}$ waves (QRS) complex on ECG signals are segmented based on k-means clustering and tracking local extrema points. Feature extraction and selection are then performed, and a dataset is created by calculating measurement parameters for each QRS points separately. Training sets and test sets based on 8-fold cross validation are generated. A hybrid model based on machine learning models including decision tree (DT), k-nearest neighbor (KNN), random forest (RF), naïve bayes (NB), linear discriminant analysis (LDA), support vector machines (SVM) and quadratic discriminant analysis (QDA) is developed to classify cardiovascular diseases (CVD) into 7 different classes such as normal sinus rhythm (NSR), atrial premature beat (APB), atrial fibrillation (AFIB), premature ventricular contraction (PVC), ventricular bigeminy (VB), left bundle branch block beat (LBBBB) and right bundle branch block beat (RBBBB). Sensitivity, specificity, accuracy, and Matthews correlation coefficient (MCC) of detection of QRS complex are obtained respectively as $94.75 \%, 95.96 \%, 95.57 \%$ and 0.90 . Sensitivity, specificity, accuracy and MCC of classification of CVD classes are obtained respectively as $92.33 \%, 92.50 \%$, $92.41 \%, 0.85$.
\end{abstract}

Keywords: CVD, ECG signal, machine learning, signal processing.

\section{Introduction}

Cardiovascular diseases (CVD) come first all around the world according to World Health Organization (WHO) statistics [1]. Even though preventions have been taken as much as possible in order to save people's lives, estimations conclude that up to $90 \%$ of CVD can be preventable [2].

Electrocardiography (ECG) is a basic, accessible, and noninvasive method used for detection of CVD. ECG graphically records electrical activities of hearts during systole and diastole. The fact that ECG uncovers substantially important information about hearts in a simple, fast, and non-invasive ways brings about growing use of it [1,2].

$\mathrm{P}$ wave, $\mathrm{Q}$ wave, $\mathrm{R}$ wave, $\mathrm{S}$ wave and $\mathrm{T}$ wave stand for $\mathrm{P}, \mathrm{Q}, \mathrm{R}, \mathrm{S}$ and $T$ points of PQRST complex on ECG signal fragments. PQRST complex corresponding to each heartbeat are observed throughout each heartbeat. Schematic representation of PQRST complex is shown in Fig. 1.

PQRST complex is such an informative clue that many studies in the literature analyse PQRST complex instead of long-duration ECG signal $[3,4]$. The most developed topics related to ECG signal analysis in the state of art are classified as ECG beat detection and

\footnotetext{
'Medical Engineering, Karabuk University, Karabük-78050, TURKEY ORCID ID: 0000-0003-0511-1933

${ }^{2}$ Medicine Faculty, Karabuk University, Karabük - 78050, TURKEY ORCID ID: 0000-0002-1086-9514

* Corresponding Author Email: eftalsehirli@karabuk.edu.tr
}

classification [4-7], deep learning [4,8-10], principal component analysis [4,11,12], higher order statistics [4,13,14], feature selection and dimensionality reduction [4,15-18], noise [4,19-21], discrete wavelet transform $[4,11,20]$, independent component analysis [4,11] and machine learning [4,19,23-25].

Fig. 1. Schematic representation of PQRST complex.

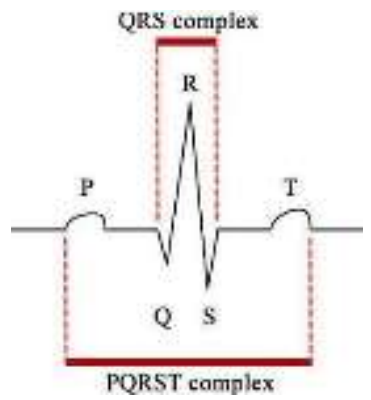

Inspired by these works, analysis and classification, this work makes a six-fold contribution association with ECG signal processing, analysis, and classification:

1. A novel method based on denoising filters, consecutive difference method, pixel tracking and k-means clustering method is developed to detect locations of R peaks on ECG signals.

2. A hybrid model based on machine learning models is developed to classify CVD on ECG signals into 7 different classes such as normal sinus rhythm (NSR), atrial premature beat (APB), 
atrial fibrillation (AFIB), premature ventricular contraction (PVC), ventricular bigeminy (VB), left bundle branch block beat (LBBBB) and right bundle branch block beat (RBBBB)

3. Textural features appeared in the literature some of which are standard deviation, mean value, median value, energy value, correlation value, homogeneity value [26] based on $\mathrm{x}$ and $\mathrm{y}$ coordinates of $\mathrm{QRS}$ points, contrast value based on $\mathrm{x}$ coordinates of QRS points and pulse are calculated separately for feature extraction and selection. Hence, a 837x 40 dataset is created based on these measurement features. Subsequently, training sets and test sets are created based on 8 -fold cross validation.

4. Although $P$ and $T$ waves carry information on ECG signal analysis in medicine, ECG signals are analyzed without using $\mathrm{P}$ and $\mathrm{T}$ waves, PR segment, PR interval, ST segment and QT interval in this work.

5. Automated detection of QRS complex and classification of CVD on ECG signals with a high sensitivity, specificity, and accuracy as fast as possible are realized in this work.

6 . The main contribution of the study is to control and identify emergency cases using a hybrid system based on machine learning and present results for healthcare staff. This creates time for healthcare staff to evaluate ECG signal changes in a fast and accurate ways.

\section{Materials}

In this work, the MIT-BIH arrhythmia dataset compatible with Matlab and reached at Physio.net (http://www.physionet.org) is utilized in order to obtain ECG signals [27,28]. In this dataset, there are 1000 ECG signal fragments taken from 45 patients whose ages range between 23 and 89 and 17 different classes with normal sinus rhythm, pacemaker rhythm and 15 types of cardiac dysfunctions. However, 837 ECG signal fragments and 7 different classes are used in this work since other classes are not sufficient and qualified from the standpoint of machine learning models, training sets and test sets. Hence, 163 ECG signal fragments are excluded. All ECG signals were recorded at a sampling frequency of $360 \mathrm{~Hz}$ and gain of $200 \mathrm{adu} / \mathrm{mV}$. Each signal contains $10-\mathrm{s}$ samples. Each signal is $3600 \times 1$ in size [4]. A description about this dataset including ECG signals is shown in Table 1.

Table 1. A description about the MIT-BIH arrhythmia dataset including ECG signals [4]

\begin{tabular}{cc}
\hline Class & Number of fragments \\
\hline NSR & 283 \\
APB & 66 \\
AFIB & 135 \\
PVC & 133 \\
VB & 55 \\
LBBBB & 103 \\
RBBBB & 62 \\
Total & 837 \\
\hline
\end{tabular}

NSR is a class name that heart works normally. APB is an abnormal contraction of the heart after an electrical conduction from the atrium before the ventricles contract normally. It generally occurs in conditions such as hypoxia, anxiety, digital intoxication. In AFIB, instead of the normal contraction of the atria, it starts to tremble as a result of rapid and abnormal electrical stimulation. In general, these beats pass to the ventricles, increasing the heart rhythm and the risk of embolism. PVC is the abnormal contraction of a ventricle as a result of abnormal electrical stimulation from the ventricle. It occurs due to reasons such as excess adrenaline, increased calcium concentration and cardiomiopathies. VB is a cardiac arrhythmia in which there is a single ectopic beat or an irregular heartbeat following each regular heartbeat. Often this is due to ectopic beats that occur frequently enough to be one after each sinus beat. Electrolyte imbalance occurs in situations such as hypoxia. LBBBB and RBBBB are caused by a delay in the electrical conduction paths during the heartbeat, on the right and left. In this case, the heart has difficulty pumping regular and sufficient blood to the body. We also encounter situations such as cardiomypathy, heart muscle infections, heart attacks, hypoxia, and embolism [29].

A software program is developed using Matlab 2018a program via a computer containing $4.00 \mathrm{GHz}-\mathrm{i} 7 \mathrm{CPU}$ and $32 \mathrm{~GB}$ RAM.

837 ECG signal fragments were evaluated by a clinical expert. All QRS points and CVD were manually marked. Sensitivity, specificity, accuracy, F1 score and MCC values of segmentation of QRS complex and classification of CVD are calculated and compared with clinical expert.

\section{Methods}

The developed method includes four stages as pre-processing, segmentation, postprocessing and analysis. ECG signals are made ready for segmentation during the pre-processing stage. QRS complex are segmented on ECG signals during segmentation stage. Measurement parameters related to QRS complex are calculated and a dataset for machine learning methods is created during postprocessing stage. Classification of ECG signals are performed during analysis stage. The flow chart of the developed method in this work is shown in Fig. 2.

As it can be from the flow chart, an ECG signal is loaded to the program as an input signal. In the pre-processing stage, wavelet signal denoising method [30], moving average filter [31] and zero phase filter [32] are respectively applied on the input ECG signal to remove noises and smooth baseline. While wavelet signal denoising method is performed with an 11-window size, moving average filter and zero phase filter are applied with a 9-window size. Window sizes are empirically preferred. Consecutive difference method [33] is then applied in order to uncover transition between points and find possible all local maximum points in an accurate way. Mathematical descriptions of methods are given in equations 1-4.

$s(n)=f(n)+\sigma e(n)$

Where f represents input ECG signal, s represents output ECG signal, $\mathrm{n}$ takes value between 0 and length of signal, e(n) is Gaussian random variables between 0 and 1 and $\sigma$ is variance [30].

$y[i]=\frac{1}{M} \sum_{j=0}^{M-1} x[i+j]$

Where $\mathrm{M}$ is moving window size, $\mathrm{x}$ represents input ECG signal, $y$ represents output ECG signal, i represents $x$ coordinate of ECG signal and $\mathrm{j}$ controls $\mathrm{x}$ coordinates in window [31].

$Y\left(e^{j \omega}\right)=X\left(e^{j \omega}\right) H\left(e^{j \omega}\right)$

Where $X\left(e^{j \omega}\right)$ and $H\left(e^{j \omega}\right)$ are the Fourier transforms of $\mathrm{x}[\mathrm{n}]$ and $\mathrm{h}[\mathrm{n}]$ respectively. $x[n]$ is input ECG signal and $h[n]$ is the filter's impulse response. A zero-phase filter is a special case of a linearphase filter in which the phase slope is 0 [32].

$y[i]=\sum_{i=0}^{L-1} x[i+1]-x[i]$

Where $\mathrm{L}$ is length of ECG signal, $\mathrm{x}$ represents input ECG signal, $\mathrm{y}$ represents output ECG signal and i represents $\mathrm{x}$ coordinate of ECG 
signal [33].

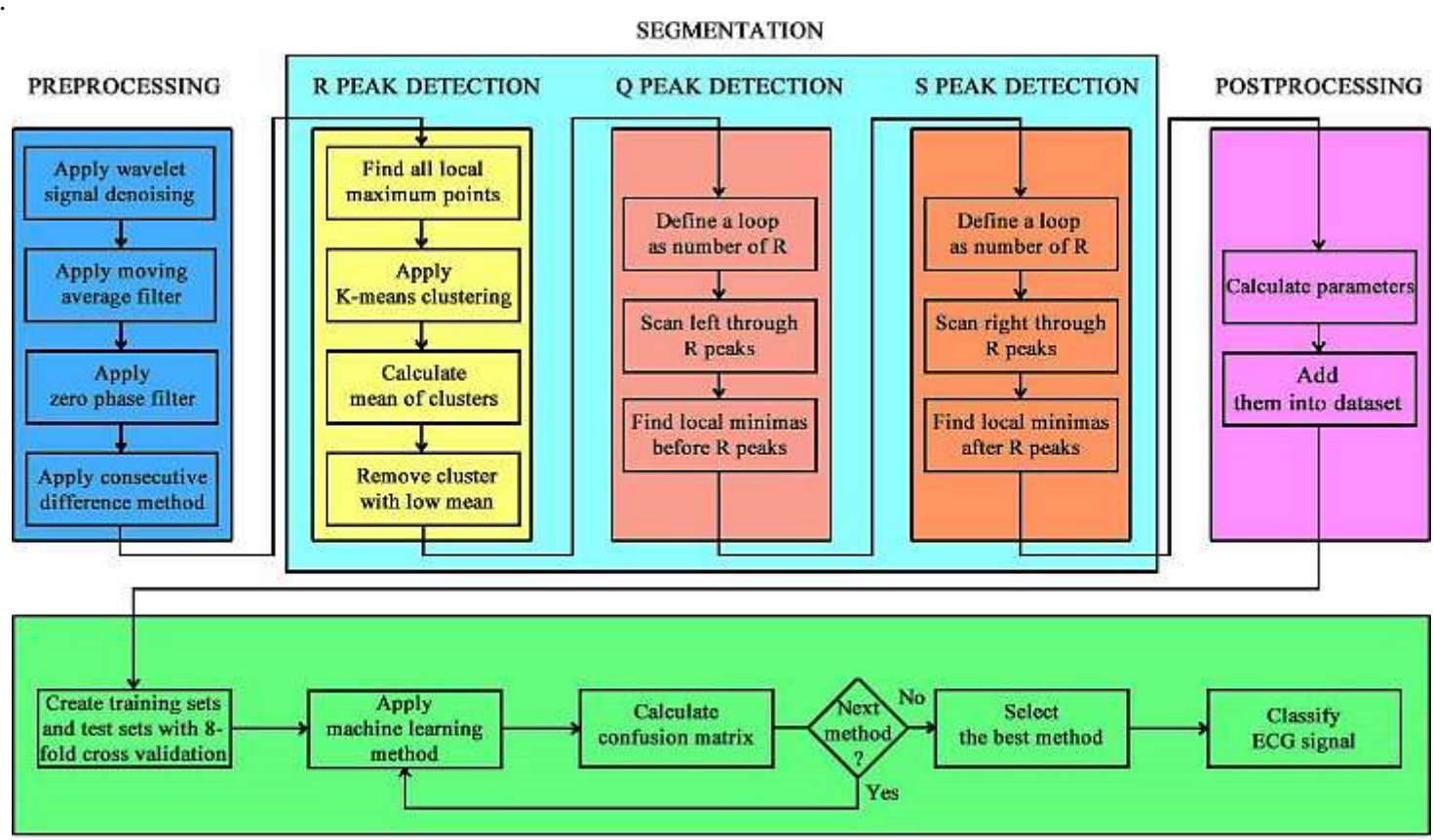

ANALYSIS

Fig. 2. The flow chart of the developed method.

Segmentation stage mainly focuses on $\mathrm{R}$ peak detection. Knowing that $\mathrm{R}$ peaks locate at local maximum points of ECG signals, it is focused on detecting local maximum points on corrected signal. According to the pseudocode in Algorithm 1 and maximum point representation given in Fig. 3, all local maximum points standing for candidate $\mathrm{R}$ peaks are found.

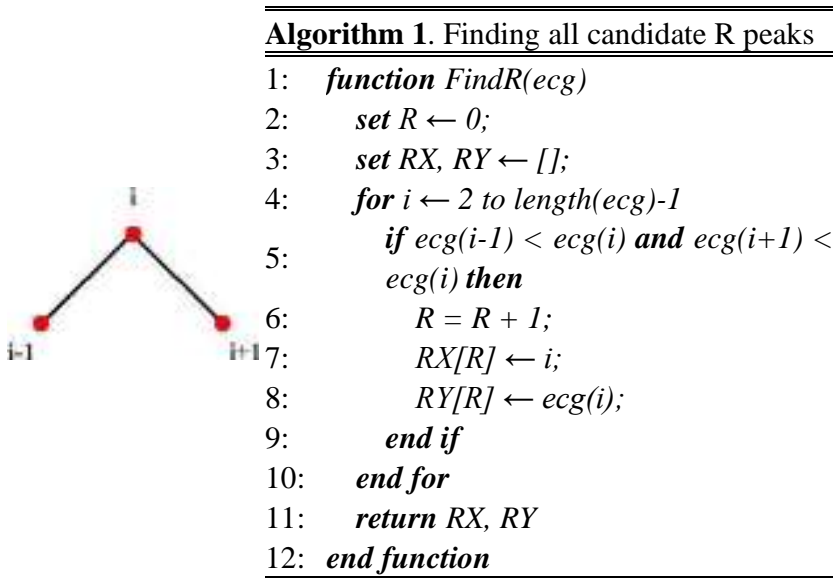

Fig. 3. Detection of all candidate R peaks.

Where RX represents $\mathrm{x}$ coordinates of $\mathrm{R}$ peaks and RY represents $\mathrm{y}$ coordinates of $\mathrm{R}$ peaks.

Although all local maximum points are detected on ECG signal fragments, all of them are not R peaks of ECG signals. k-means clustering method is utilized to eliminate false-positive candidate $\mathrm{R}$ peaks and obtain true-positive $\mathrm{R}$ peaks among all local maximum points. The fact that $\mathrm{K}$ is selected as 2 separates local maximum points such as true-positive $\mathrm{R}$ peaks and false positive $\mathrm{R}$ peaks. While one cluster represents local maximum points whose mean value is low, another cluster represents local maximum points whose mean value is high. Since cluster with low mean value consists of false-positive $\mathrm{R}$ peaks, this cluster is removed. As a result, true $\mathrm{R}$ peaks are detected, $\mathrm{x}$ and $\mathrm{y}$ coordinates of them are stored in a list.
With the detection of $\mathrm{R}$ peaks, definition of QRS complex shown in Fig. 1 is utilized. Q points of QRS complex are identified by scanning left through all $\mathrm{R}$ peaks. When a point at the left direction of $\mathrm{R}$ peak is lower than two points before and after it, this point is hold in $\mathrm{Q}$ list. The pseudocode in Algorithm 2 and $\mathrm{Q}$ point representation are given in Fig. 4.

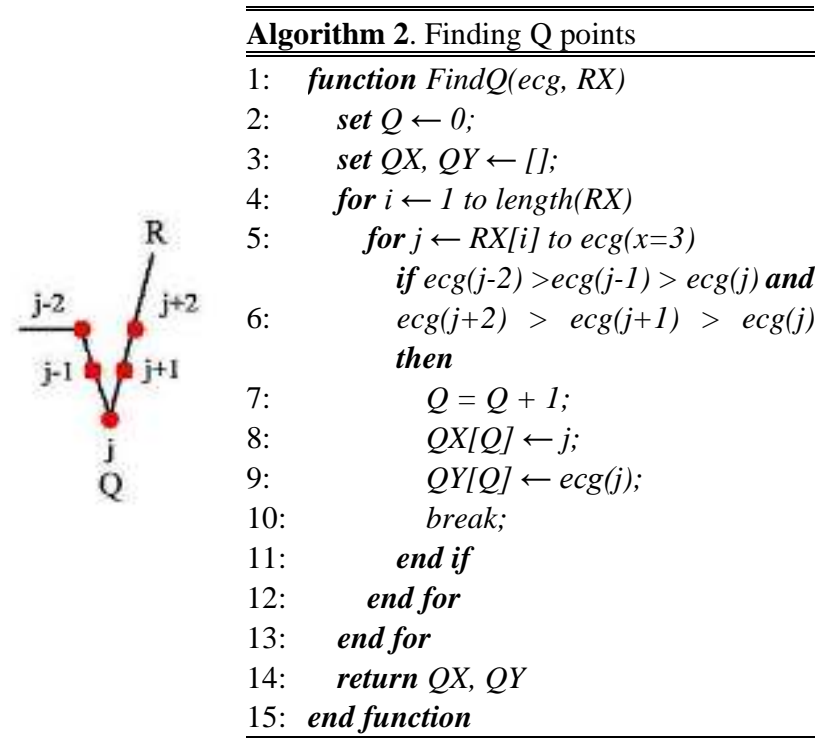

Fig. 4. Detection of Q points.

Where QX represents x coordinates of Q points and QY represents $\mathrm{y}$ coordinates of $\mathrm{Q}$ points.

In the same way as identification of Q points is performed, $S$ points of QRS complex are identified by scanning right through all $\mathrm{R}$ peaks. When a point at the right direction of $\mathrm{R}$ peak is lower than two points before and after it, this point is hold in $\mathrm{S}$ list. The pseudocode in Algorithm 3 and $\mathrm{S}$ point representation are given in Fig. 5. 


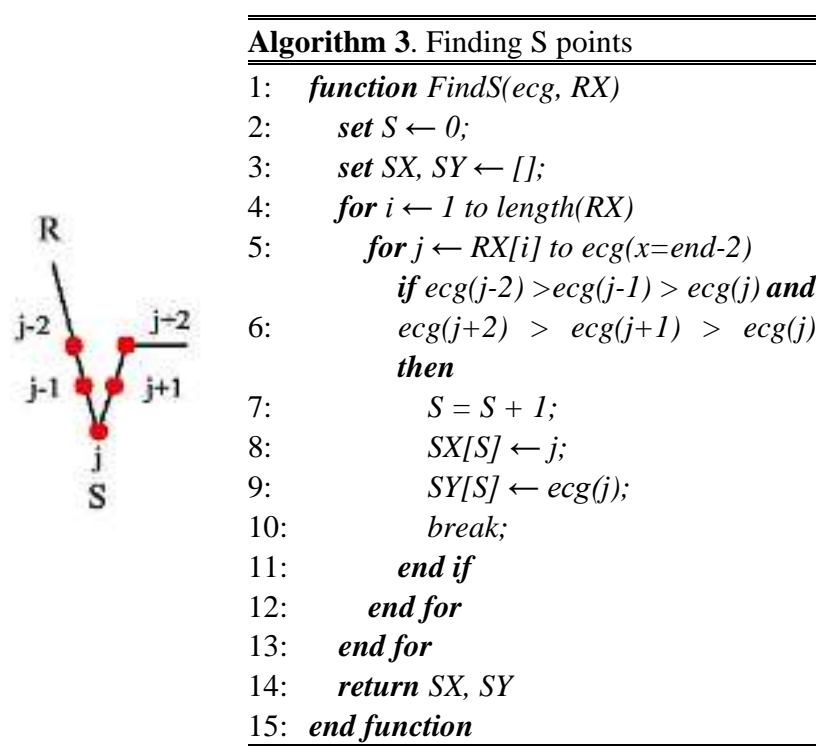

Fig. 5. Detection of S points.

Where SX represents $\mathrm{x}$ coordinates of S points and SY represents $y$ coordinates of $S$ points.

After detection of $\mathrm{x}$ and $\mathrm{y}$ coordinates of each QRS complex, feature extraction is performed to create a dataset for machine learning models in the postprocessing stage. Standard deviation, mean, median, energy, entropy and homogeneity based on $\mathrm{x}$ and $\mathrm{y}$ coordinates, contrast based on $\mathrm{x}$ coordinates of QRS points and pulse value are calculated separately. Thus, an $837 \times 40$ dataset is created as a result of analysis of 837 ECG signal fragments. Equations 5-11 show the mathematical description of features [26].

Standard deviation $=\sqrt{\frac{1}{N} \sum_{i=1}^{N}\left(x_{i}-\mu\right)^{2}}$

Where $\mathrm{N}$ is total number, $\mu$ is mean and $x_{i}$ is $\mathrm{i}$-th individual value.

Mean $=\frac{1}{N} \sum_{i=1}^{N} x_{i}$

Where $\mathrm{N}$ is the total number, $x_{i}$ is the $\mathrm{i}$-th individual value and $\mu$ is the mean value.

Median $=x_{\frac{N+1}{2}}$

Where $\mathrm{N}$ is the total number and $\mathrm{x} \frac{N+1}{2}$ - is $\frac{N+1}{2}$-th value.

Energy $=\sum_{i=1}^{N}\left(x_{i}\right)^{2}$

Where $\mathrm{N}$ is the total number and $x_{i}$ is the $\mathrm{i}$-th individual value.

Entropy $=-\sum_{i=1}^{N} \ln \left(x_{i}\right) x_{i}$

Where $\mathrm{N}$ is the total number and $x_{i}$ is the $\mathrm{i}$-th individual value.

Homogeneity $=-\sum_{i=1}^{N} \frac{x_{i}}{1+\left(x_{i}\right)^{2}}$

Where $\mathrm{N}$ is the total number and $x_{i}$ is the $\mathrm{i}$-th individual value.

Contrast $=\sum_{i=1}^{N} x_{i}(i)^{2}$

Where $\mathrm{N}$ is the total number and $x_{i}$ is the $\mathrm{i}$-th individual value.

The dataset is divided into two sets as training set with the $70 \%$ part of the dataset and test set with the $30 \%$ part of the dataset. The validity of the hybrid method is ensured by 8 -fold cross validation. A hybrid model based on machine learning models is developed to classify CVD on ECG signals into 7 different classes such as NSR, APB, AFIB, PVC, VB, LBBBB and RBBBB. Machine learning models with their attributes and settings are presented in Table 2.
Table 2. Machine learning models with their attributes and settings.

\begin{tabular}{ll}
\hline Model & Attributes and settings \\
\hline DT & C4.5 learning method is used. \\
KNN & K is identified as 2 which means class and others. \\
RF & The number of decision trees is identified as 100. \\
NB & The distribution parameter is identified as "Gaussian". \\
LDA & The discriminant type is identified as "Linear". \\
SVM & The kernel is designated as "Gaussian". \\
QDA & The discriminant type is identified as "Quadratic". \\
\hline
\end{tabular}

According to the developed hybrid model, training sets and test sets of machine learning models are created again and again by focusing on only one class. Other classes are called as nonclassified. During training of NSR, 50\% of training set and test set are composed of NSR. Another 50\% of training set and test set are composed of AFIB, PVC, LBBBB, APB, RBBBB and VB. During training of AFIB, $50 \%$ of training set and test set are composed of AFIB. Another $50 \%$ of training set and test set are composed of PVC, LBBBB, APB, RBBBB and VB. During training of PVC, $50 \%$ of training set and test set are composed of PVC. Another $50 \%$ of training set and test set are composed of LBBBB, APB, RBBBB and VB. During training of LBBBB, $50 \%$ of training set and test set are composed of LBBBB. Another $50 \%$ of training set and test set are composed of $\mathrm{APB}, \mathrm{RBBBB}$ and VB. During training of $\mathrm{LBBBB}, 50 \%$ of training set and test set are composed of LBBBB. Another $50 \%$ of training set and test set are composed of APB, RBBBB and VB. During training of APB, $50 \%$ of training set and test set are composed of APB. Another 50\% of training set and test set are composed of RBBBB and VB. During training of RBBBB and VB, $50 \%$ of training set and test set are composed of VB. Another $50 \%$ of training set and test set are composed of RBBBB.

Confusion matrix is used to summarize and validate results. It mainly consists of four parameters such as True Positive (TP), False Positive (FP), False Negative (FN) and True Negative (TN). TP stands for correctly identified conditions. FP stands for incorrectly identified conditions. FN stands for incorrectly rejected conditions. TN stands for correctly rejected conditions. Mathematical descriptions of sensitivity, specificity, accuracy, F1 score and MCC are given in Equations 8-12 [34,35].

?

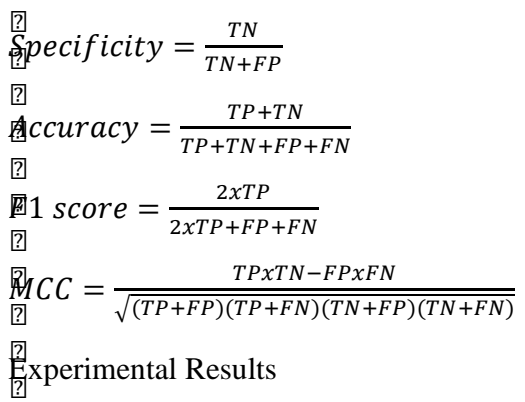

In this work, 837 ECG signal fragments having 3600x1 in size with different classes such as NSR, APB, AFIB, PVC, VB, LBBBB and RBBBB are analysed. Pre-processing stage is firstly applied on ECG signals. Part of one sample original ECG signal and the btained ECG signal after pre-processing are shown in Fig. 6.

En the segmentation stage, $x$ and $y$ coordinates of QRS complex are glentified and segmented. One sample ECG signal for each class Whose QRS complex are marked are shown in Fig. 7.

回

$=$

International Journal of Intelligent Systems and Applications in Engineering 
(a)

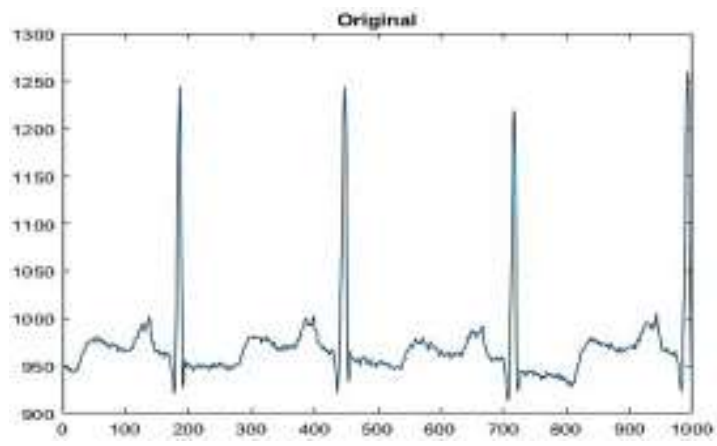

(b)

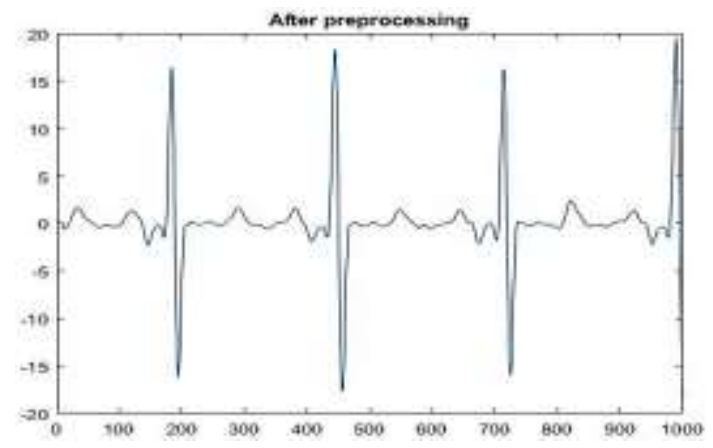

Fig. 6. Part of sample NSR ECG signal. a) Original, b) After preprocessing.

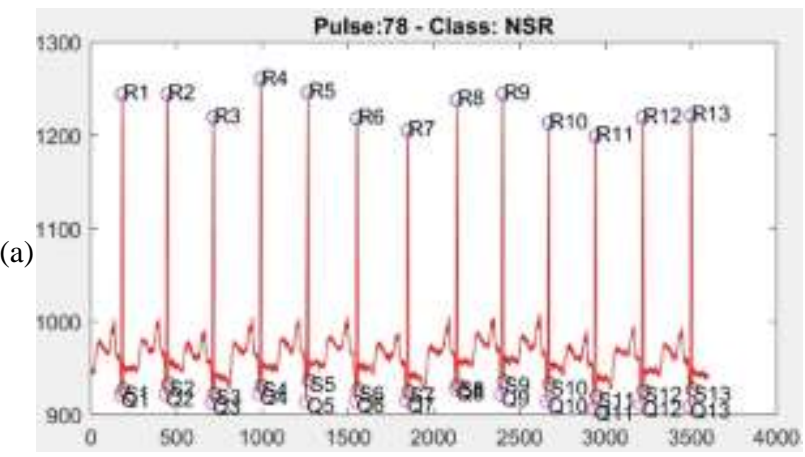

(b)
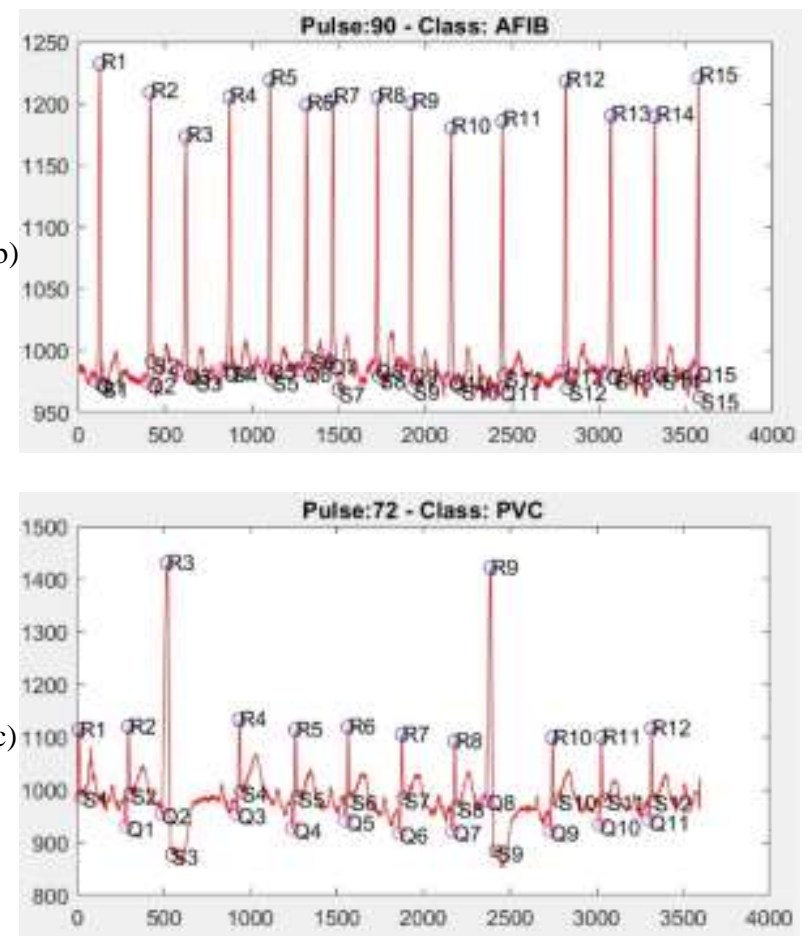

(d)
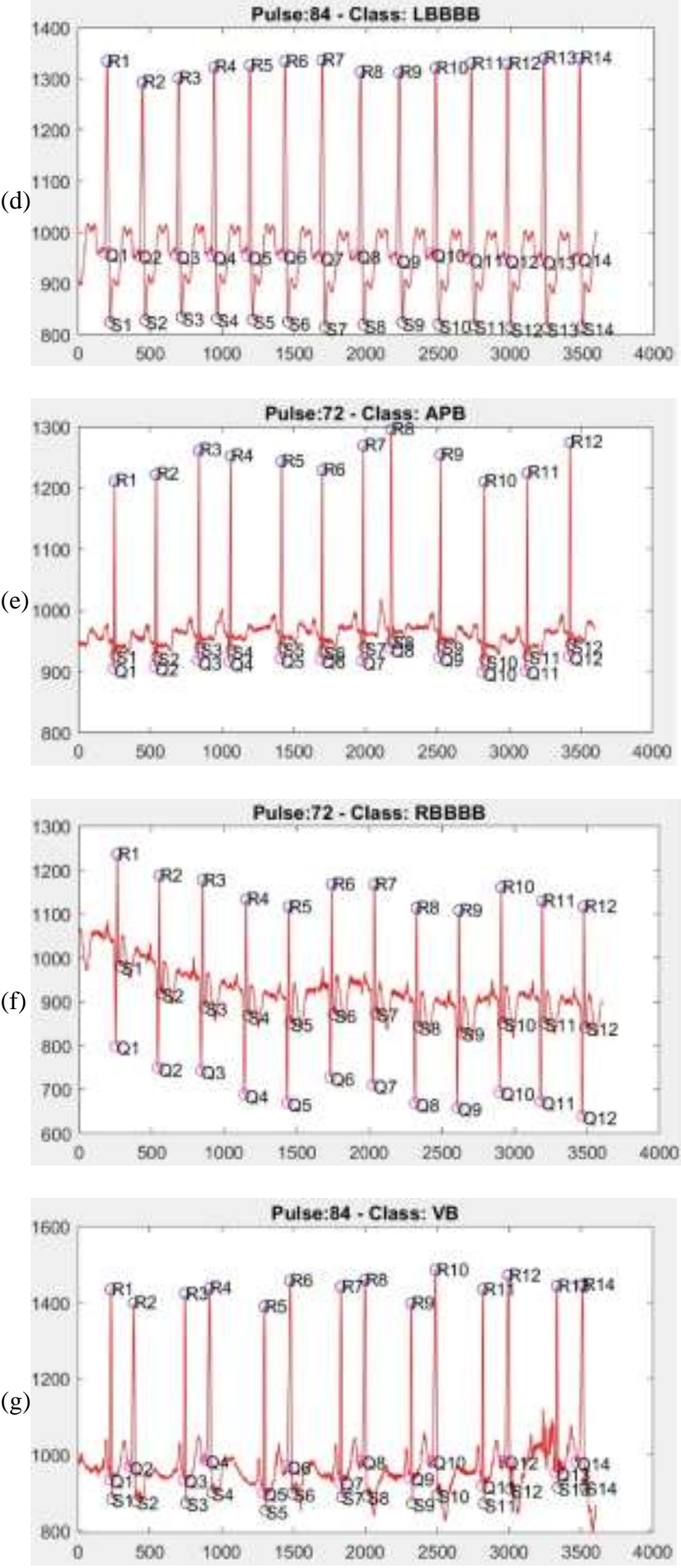

Fig. 7. ECG signals whose QRS complex are marked. a) NSR, b) AFIB, c) PVC, d) LBBBB, e) APB, f) RBBBB, g) VB.

Performance metrics as sensitivity, specificity, accuracy, F1 score and MCC parameters related to segmentation of QRS complex (Q peak detection, $\mathrm{R}$ peak detection and $\mathrm{S}$ peak detection method) are calculated and shown in Table 3 .

Standard deviation, mean, median, energy, entropy and homogeneity based on $\mathrm{x}$ and $\mathrm{y}$ coordinates, contrast based on $\mathrm{x}$ coordinates of QRS complex and pulse value are calculated separately for each ECG signal. Thus, an 837x40 dataset is created. The dataset is separated into training sets and test sets. A description about training set and test set for each ECG class is shown in Table 4. 
Table 3. QRS complex segmentation performance.

\begin{tabular}{|c|c|c|c|c|c|c|}
\hline CVD & Wave & $\begin{array}{c}\text { Sensitivity } \\
(\%)\end{array}$ & $\begin{array}{c}\text { Specificity } \\
(\%)\end{array}$ & $\begin{array}{c}\text { Accuracy } \\
(\%)\end{array}$ & $\begin{array}{c}\text { F1 score } \\
(\%)\end{array}$ & MCC \\
\hline \multirow{3}{*}{$\begin{array}{l}\frac{1}{n} \\
Z\end{array}$} & $R$ & 99.91 & 96.57 & 97.66 & 96.55 & 0.95 \\
\hline & $Q$ & 85.96 & 93.46 & 91.08 & 85.96 & 0.79 \\
\hline & $\widetilde{S}$ & 95.89 & 98.00 & 97.30 & 95.89 & 0.94 \\
\hline \multirow{3}{*}{$\stackrel{\varphi}{\frac{1}{4}}$} & $R$ & 99.39 & 99.90 & 99.73 & 99.59 & 0.99 \\
\hline & $Q$ & 90.35 & 95.41 & 93.78 & 90.35 & 0.86 \\
\hline & $S$ & 94.06 & 97.13 & 96.13 & 94.06 & 0.91 \\
\hline \multirow{3}{*}{$\sum_{2}^{u}$} & $R$ & 97.77 & 96.77 & 97.10 & 95.72 & 0.94 \\
\hline & $Q$ & 100.00 & 100.00 & 100.00 & 100.00 & 1.00 \\
\hline & S & 99.36 & 99.68 & 99.58 & 99.36 & 0.99 \\
\hline \multirow{3}{*}{$\begin{array}{l}m \\
m \\
m \\
m\end{array}$} & $R$ & 97.13 & 83.26 & 87.45 & 82.35 & 0.75 \\
\hline & $Q$ & 94.63 & 97.39 & 96.48 & 94.63 & 0.92 \\
\hline & $\tilde{S}$ & 78.59 & 90.37 & 86.71 & 78.59 & 0.69 \\
\hline \multirow{3}{*}{$\frac{\infty}{2}$} & $R$ & 100.00 & 99.95 & 99.96 & 99.95 & 0.99 \\
\hline & $Q$ & 95.11 & 97.61 & 96.79 & 95.11 & 0.93 \\
\hline & S & 99.68 & 99.84 & 99.79 & 99.68 & 0.99 \\
\hline \multirow{3}{*}{$\begin{array}{l}n \\
n \\
n \\
\underline{n}\end{array}$} & $R$ & 100.00 & 87.94 & 91.63 & 87.95 & 0.84 \\
\hline & $Q$ & 94.99 & 97.55 & 96.71 & 94.99 & 0.94 \\
\hline & $\tilde{S}$ & 87.23 & 94.07 & 91.88 & 87.29 & 0.81 \\
\hline \multirow{3}{*}{$\stackrel{\infty}{>}^{\infty}$} & $R$ & 91.05 & 95.31 & 93.84 & 91.05 & 0.86 \\
\hline & $Q$ & 88.37 & 94.70 & 92.72 & 88.37 & 0.83 \\
\hline & S & 95.02 & 97.66 & 96.81 & 95.02 & 0.93 \\
\hline \multirow{3}{*}{ 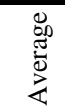 } & $R$ & 98.39 & 94.73 & 95.92 & 94.02 & 0.91 \\
\hline & $Q$ & 92.32 & 96.32 & 95.03 & 92.32 & 0.89 \\
\hline & $S$ & 93.45 & 96.86 & 95.75 & 93.46 & 0.90 \\
\hline
\end{tabular}

Table 4. Description of training set and test set of each ECG classes.

\begin{tabular}{cccc}
\hline CVD & $\begin{array}{c}\text { Size of } \\
\text { fragment }\end{array}$ & $\begin{array}{c}\text { Size of } \\
\text { training set }\end{array}$ & $\begin{array}{c}\text { Size of } \\
\text { test set }\end{array}$ \\
\hline NSR & $283 \times 40$ & $396 \times 40$ & $170 \times 40$ \\
AFIB & $135 \times 40$ & $188 \times 40$ & $82 \times 40$ \\
PVC & $133 \times 40$ & $186 \times 40$ & $80 \times 40$ \\
LBBBB & $103 \times 40$ & $144 \times 40$ & $62 \times 40$ \\
APB & $66 \times 40$ & $92 \times 40$ & $40 \times 40$ \\
RBBBB & $62 \times 40$ & $78 \times 40$ & $32 \times 40$ \\
VB & $55 \times 40$ & $78 \times 40$ & $32 \times 40$ \\
\hline
\end{tabular}

A hybrid model based on machine learning models is developed to classify CVD such as NSR, AFIB, PVC, LBBBB, APB, RBBBB and VB. According to training sets and test sets at Table 4, one class is selected for classification and other classes are collected in another class. With the 8-fold cross validation, performance metrics of each CVD are calculated and presented in Table 5.

Performance metrics of hybrid model to classify ECG classes are calculated as $92.33 \%$ sensitivity, $92.50 \%$ specificity, $92.41 \%$ accuracy, $92.42 \%$ F1 score and 0.85 for MCC. According to performance metrics, the proposed hybrid model based on machine learning models to classify CVD is identified and shown in Fig. 8. Analysis and classification of an ECG signal takes 0.31 seconds in average in a full-automatedly way via the developed program.

Table 5. Performance metrics of classification of CVD.

\begin{tabular}{|c|c|c|c|c|c|c|c|c|}
\hline CVD & $\begin{array}{l}\text { Performance } \\
\text { metric }\end{array}$ & DT & KNN & RF & NB & LDA & SVM & QDA \\
\hline NSR & $\begin{array}{l}\text { Sensitivity } \\
\text { Specificity } \\
\text { Accuracy } \\
\text { F1 score } \\
\text { MCC }\end{array}$ & $\begin{array}{c}92.62 \\
86.27 \\
89.45 \\
89.74 \\
0.79\end{array}$ & $\begin{array}{c}80.74 \\
78.07 \\
79.41 \\
79.61 \\
0.59\end{array}$ & $\begin{array}{c}94.26 \\
88.73 \\
91.50 \\
91.76 \\
0.83\end{array}$ & $\begin{array}{c}92.10 \\
75.12 \\
83.71 \\
85.14 \\
0.68\end{array}$ & $\begin{array}{c}89.96 \\
82.38 \\
86.17 \\
86.64 \\
0.73\end{array}$ & $\begin{array}{c}53.69 \\
94.67 \\
74.18 \\
67.36 \\
0.53\end{array}$ & $\begin{array}{c}89.14 \\
81.56 \\
85.35 \\
85.83 \\
0.71\end{array}$ \\
\hline AFIB & $\begin{array}{l}\text { Sensitivity } \\
\text { Specificity } \\
\text { Accuracy } \\
\text { F1 score } \\
\text { MCC }\end{array}$ & $\begin{array}{c}87.80 \\
91.77 \\
89.79 \\
89.57 \\
0.80\end{array}$ & $\begin{array}{c}84.45 \\
80.49 \\
82.47 \\
82.86 \\
0.66\end{array}$ & $\begin{array}{c}90.55 \\
93.90 \\
92.23 \\
92.07 \\
0.85\end{array}$ & $\begin{array}{l}84.15 \\
66.46 \\
75.30 \\
77.29 \\
0.52\end{array}$ & $\begin{array}{c}92.68 \\
79.88 \\
86.28 \\
87.13 \\
0.73\end{array}$ & $\begin{array}{c}57.93 \\
97.87 \\
77.90 \\
71.72 \\
0.61\end{array}$ & $\begin{array}{c}76.83 \\
97.26 \\
87.04 \\
85.40 \\
0.76\end{array}$ \\
\hline PVC & $\begin{array}{l}\text { Sensitivity } \\
\text { Specificity } \\
\text { Accuracy } \\
\text { F1 score } \\
\text { MCC }\end{array}$ & $\begin{array}{c}87.81 \\
92.19 \\
90.00 \\
89.75 \\
0.80\end{array}$ & $\begin{array}{c}78.13 \\
79.38 \\
78.75 \\
78.56 \\
0.58\end{array}$ & $\begin{array}{c}88.75 \\
92.50 \\
90.63 \\
90.40 \\
0.82\end{array}$ & $\begin{array}{c}80.31 \\
75.63 \\
77.97 \\
78.48 \\
0.56\end{array}$ & $\begin{array}{c}68.44 \\
88.44 \\
78.44 \\
76.03 \\
0.58\end{array}$ & $\begin{array}{c}97.81 \\
47.19 \\
72.50 \\
78.07 \\
0.52\end{array}$ & $\begin{array}{c}91.25 \\
78.75 \\
85.00 \\
85.89 \\
0.71\end{array}$ \\
\hline LBBBB & $\begin{array}{l}\text { Sensitivity } \\
\text { Specificity } \\
\text { Accuracy } \\
\text { F1 score } \\
\text { MCC }\end{array}$ & $\begin{array}{c}91.94 \\
92.34 \\
92.14 \\
92.10 \\
0.84\end{array}$ & $\begin{array}{c}87.90 \\
79.84 \\
83.87 \\
84.48 \\
0.68\end{array}$ & $\begin{array}{c}89.54 \\
97.97 \\
93.75 \\
93.41 \\
0.88\end{array}$ & $\begin{array}{c}54.84 \\
85.48 \\
70.16 \\
62.60 \\
0.45\end{array}$ & $\begin{array}{c}98.79 \\
93.55 \\
96.17 \\
96.33 \\
0.93\end{array}$ & $\begin{array}{c}46.37 \\
99.19 \\
72.78 \\
62.89 \\
0.54\end{array}$ & $\begin{array}{c}94.76 \\
94.76 \\
94.76 \\
94.73 \\
0.90\end{array}$ \\
\hline APB & $\begin{array}{l}\text { Sensitivity } \\
\text { Specificity } \\
\text { Accuracy } \\
\text { F1 score } \\
\text { MCC }\end{array}$ & $\begin{array}{c}89.38 \\
93.75 \\
91.56 \\
91.36 \\
0.83\end{array}$ & $\begin{array}{c}85.63 \\
81.25 \\
83.44 \\
83.78 \\
0.67\end{array}$ & $\begin{array}{c}88.13 \\
93.13 \\
90.63 \\
90.38 \\
0.82\end{array}$ & $\begin{array}{l}88.75 \\
58.75 \\
73.75 \\
77.12 \\
0.51\end{array}$ & $\begin{array}{c}81.25 \\
73.75 \\
77.50 \\
78.36 \\
0.55\end{array}$ & $\begin{array}{c}51.25 \\
95.63 \\
73.44 \\
65.75 \\
0.53\end{array}$ & $\begin{array}{c}64.38 \\
93.75 \\
79.06 \\
74.69 \\
0.62\end{array}$ \\
\hline RBBBB & $\begin{array}{l}\text { Sensitivity } \\
\text { Specificity } \\
\text { Accuracy } \\
\text { F1 score } \\
\text { MCC }\end{array}$ & $\begin{array}{c}95.31 \\
94.53 \\
94.92 \\
94.98 \\
0.90\end{array}$ & $\begin{array}{c}77.34 \\
92.19 \\
84.77 \\
83.64 \\
0.71\end{array}$ & $\begin{array}{c}96.88 \\
92.97 \\
94.92 \\
95.10 \\
0.90\end{array}$ & $\begin{array}{l}82.81 \\
60.16 \\
71.48 \\
74.47 \\
0.45\end{array}$ & $\begin{array}{c}83.59 \\
93.75 \\
88.67 \\
88.04 \\
0.78\end{array}$ & $\begin{array}{c}100.00 \\
58.59 \\
79.30 \\
83.07 \\
0.64\end{array}$ & $\begin{array}{l}\text { NA } \\
\text { NA } \\
\text { NA } \\
\text { NA } \\
\text { NA }\end{array}$ \\
\hline VB & $\begin{array}{l}\text { Sensitivity } \\
\text { Specificity } \\
\text { Accuracy } \\
\text { F1 score } \\
\text { MCC }\end{array}$ & $\begin{array}{c}94.53 \\
95.31 \\
94.92 \\
94.98 \\
0.90\end{array}$ & $\begin{array}{l}92.19 \\
77.34 \\
84.77 \\
83.64 \\
0.71\end{array}$ & $\begin{array}{c}92.97 \\
96.88 \\
94.92 \\
95.10 \\
0.90\end{array}$ & $\begin{array}{c}60.16 \\
82.81 \\
71.48 \\
74.47 \\
0.45\end{array}$ & $\begin{array}{c}93.75 \\
83.59 \\
88.67 \\
88.04 \\
0.78\end{array}$ & $\begin{array}{c}58.59 \\
100.00 \\
79.30 \\
83.07 \\
0.64\end{array}$ & $\begin{array}{l}\text { NA } \\
\text { NA } \\
\text { NA } \\
\text { NA } \\
\text { NA }\end{array}$ \\
\hline
\end{tabular}




\section{Conclusion and Discussion}

In this work, 837 ECG signal fragments are analyzed performing segmentation of QRS complex, feature extraction, classification of 7 different ECG classes by the proposed hybrid model based on machine learning models. A novel method based on denoising filters, consecutive difference method, pixel tracking and k-means clustering method is developed to detect locations of $\mathrm{R}$ peaks on ECG signals in an automated way. 40 different features are extracted from ECG signals mainly based on QRS complex. Thus, a dataset whose size is $837 \times 40$ is created. A developed hybrid model is trained and tested on this dataset. Even though $\mathrm{P}$ and $\mathrm{T}$ peaks are taken into consideration in medicine, only QRS complex of ECG signals are segmented instead of PQRST complex. Feature extraction is performed without using $\mathrm{P}$ and $\mathrm{T}$ peaks, PR segment, PR interval, ST segment and QT interval in this work. Automated detection of QRS complex on ECG signals and classification of 7 different ECG classes via the developed program in Matlab with high performance metrics are performed. Overall accuracy score was realized as $92.41 \%$. The proposed method for segmentation of QRS complex on ECG signals have obtained high performance metrics according to state of the art. It is quite effective as works in state of the art. Table 6 presents QRS complex segmentation works in state of the art with used dataset, methods, and results.

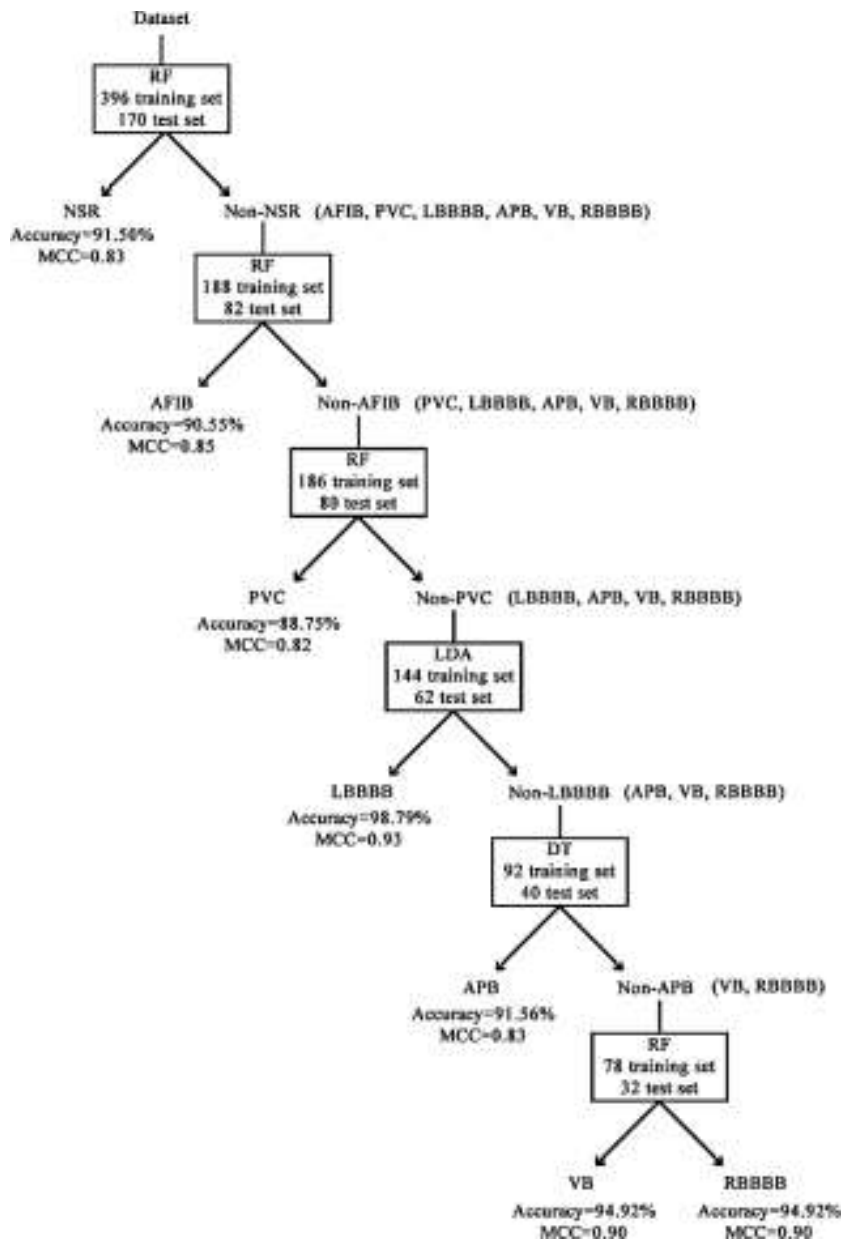

Fig. 8. The proposed hybrid model.

Table 6. QRS complex segmentation comparison [36] (WT: Wavelet transformation, DWT: Discrete wavelet transform, ACL: Anterior cruciate ligament, VLSI: Very-large-scale integration, FIR: Finite impulse response, PPV: Positive predicted value)

\begin{tabular}{llll}
\hline Author (Year) & Database & Methods & Result \\
\hline Fujita et al. (2015) [37] & MIT-BIH database & WT & $99 \%$ sensitivity \\
Di Marco et al. (2011) [38] & MIT-BIH database & WT & $99.77 \%$ sensitivity, 99.86\% PPV \\
Martinez et al. (2004) [39] & MIT-BIH database & WT & $99.8 \%$ sensitivity, 99.86\% PPV \\
Zidelmal et al. (2012) [40] & MIT-BIH database & DWT & $99.64 \%$ sensitivity, 99.82\% PPV \\
Ghaffari et al. (2011) [41] & MIT-BIH database & DWT-Based ACL & $99.94 \%$ sensitivity, 99.91\% PPV \\
Subramanian (2017) [42] & MIT-BIH database & Multiwavelet transform & $93.35 \%$ accuracy, 98.5\% sensitivity \\
& & & $97 \%$ PPV \\
Zhang et al. (2009) [43] & MIT-BIH database & Mathematical morphology & $99.81 \%$ sensitivity, 99.8\% PPV \\
Zhang et al. (2012) [44] & MIT-BIH database & Mathematical morphology, VLSI detector & $99.76 \%$ sensitivity, 99.82\% PPV \\
Curtin et al. (2018) [45] & MIT-BIH database & Windowing algorithm & $94.3 \%$ accuracy, 96\% sensitivity \\
& & & $97.3 \%$ PPV \\
Saadi et al. (2015) [46] & MIT-BIH database & FIR-Based adaptive thresholds & $99.9 \%$ sensitivity, 99.87\% PPV \\
The proposed method & MIT-BIH database & Consecutive difference method, & $95.57 \%$ accuracy, $94.75 \%$ sensitivity \\
& & k-means clustering, tracking local extreme & $95.96 \%$ specificity, $91.86 \%$ PPV \\
\hline
\end{tabular}

MIT-BIH database includes 1000 ECG signals composing of 17 CVD. The selected 7 CVD for this work composes of 837 ECG signal fragments. 163 ECG signal fragments composing of 10 CVD are excluded from this work because each excluded CVD has 16.3 ECG signals in average. This number is too low for machine learning. Thus, TN values of the developed method is balanced and memorization by machine learning models is prevented. Table 7 presents CVD classification works in state of the art with used dataset, methods, and results.

Feature extraction on dataset for machine learning models needs a clinical expert since definition and calculation of segments like ST, QT, PT require medical background. Feature extraction on ECG signals is assumed as a challenging work. The proposed method can supply feature extraction on QRS complex without needing medical background by segmenting QRS complex and calculating measurement parameters in a fully automated way.

Development of the model is simple and understandable based on complexity. The proposed method takes 0.31 seconds in average in a full-automatedly way. A comparison based on execution time could not be done because it was not shared in the literature. 
Table 7. CVD classification comparison $[47,48]$.

\begin{tabular}{|c|c|c|c|}
\hline Author (Year) & Dataset & Methods & Result \\
\hline Plawiak (2018) [49] & MIT-BIH database & $\begin{array}{l}\text { Power spectral density (using Welchs method, DFT), genetic } \\
\text { ensembles based on SVM }\end{array}$ & $\begin{array}{l}91.40 \% \text { sensitivity } \\
99.46 \% \text { specificity } \\
98.99 \% \text { accuracy }\end{array}$ \\
\hline Lu et al. (2018) [50] & MIT-BIH database & $\begin{array}{l}\text { Random over sampler algorithm, random forest, convolutional } \\
\text { neural networks }\end{array}$ & $\begin{array}{l}\text { Above } 99 \% \text { PPV, sensitivity and } \\
\text { F-score }\end{array}$ \\
\hline $\begin{array}{l}\text { Nasiri et al. } \\
(2009)[51]\end{array}$ & $\begin{array}{l}\text { MIT-BIH database } \\
\text { of } 48 \text { records }\end{array}$ & SVM and genetic algorithm & $93 \%$ accuracy \\
\hline $\begin{array}{l}\text { Dalal et al. } \\
(2016)[52]\end{array}$ & MIT-BIH database & Principle component analysis and neuro-fuzzy classifier & $96 \%$ accuracy \\
\hline $\begin{array}{l}\text { Priyadharshni et al. } \\
\text { (2015) [53] }\end{array}$ & MIT-BIH database & Improvised genetic algorithm, C4.5 and Naïve Bayes classifier & $\begin{array}{l}\text { Accuracy using } \mathrm{GA}=90 \%, \mathrm{C} 4.5 \\
=72 \% \text { and Naïve Bayes }=50 \%\end{array}$ \\
\hline
\end{tabular}

\section{Acknowledgments}

Authors thanks MIT-BIH to share ECG signal fragments.

\section{References}

[1] WHO (2014), "WHO Global status report on noncommunicable disease,"

http://apps.who.int/iris/bitstream/10665/148114/1/9789241564854 _eng.pdf?ua-1? (accessed Nov. 2020).

[2] H. C. McGill, C. A. McMahan, S. S. Gidding, "Preventing heart disease in the 21st century: implications of the Pathobiological Determinants of Atherosclerosis in Youth (PDAY) study," Circulation, vol. 117, no. 9, pp. 1216-1227, Mar. 2008.

[3] Ö. Yıldırım, P. Plawiak, R. S. Tan, U. R. Acharya, “Arrythmia detection using deep convolutional neural network with long duration ECG signals," Computers Biology and Medicine, vol. 102, pp. 411-420, Sep. 2018.

[4] P. Plawiak, "Novel methodology of cardiac health recognition based on ECG signals and evolutionary-neural system," Expert System Applications, vol. 92, pp. 334-349, Feb. 2018.

[5] P. Augustyniak, "A robust heartbeat detector nor depending on ECG sampling rate," in 2015 37th annual international conference of the IEEE Engineering in medicine and biology society (EMBC), Milan, Italy, 2015, pp. 7861-7864.

[6] R. J. Martis, U. R: Acharya, and H. Adeli, "Current methods in electrocardiogram characterization," Computers in Biology and Medicine, vol. 48, pp. 133-149, May 2014

[7] M. -H. Song, S. -P. Cho, and K. -J. Lee, "New real-time heartbeat detection method using angle of a single-lead electrocardiogram," Computers in Biology and Medicine, vol. 59, pp. 73-79, Apr. 2015.

[8] U. R. Acharya, H. Fujita, O. S. Lih, Y. Hagiwara, J. H. Tan, and M. Adam, "Automated detection of arrhytmias using different intervals of tachycardia ECG segments with convolutional neural network," Information Sciences, vol. 405, pp. 81-90, Sep. 2017.

[9] S. Kiranyaz, T. Ince, and M. Gabbouj, "Real-time patient-specific ECG classification by 1-D convolutional neural networks," IEEE Transactions on Biomedical Engineering, vol. 63, no. 3, pp. 664675, Aug. 2016.

[10] M. A. Rahhal, Y. Bazi, H. AlHichri, N. Alajlan, F. Melgani, and R. Yager, "Deep learning approach for active classification of electrocardiogram signals," Information Sciences, vol. 345, pp. 340354, Jun. 2016.

[11] F. A. Elhaj, N. Salim, A. R. Harris, T. T. Swee, and T. Ahmed, "Arrhythmia recognition and classification using combined linear and nonlinear features of ECG signals," Computer Methods and Programs in Biomedicine, vol. 127, pp. 52-63, Apr. 2016.
[12] R. Rodriguez, A. Mexicano, J. Bila, S. Cervantes, and R. Ponce, "Feature extraction of electrocardiogram signals by applying adaptive threshold and principle component analysis," Journal of Applied Research and Technology, vol. 13, no. 2, pp. 261-269, Apr. 2015.

[13] R. J. Martis, U. R. Acharya, K. Mandana, A. Ray, and C. Chakraborty, "Cardiac decision making using higher order spectra," Biomedical Signal Processing and Control, vol. 8, no. 2, pp. 193203, Mar. 2013.

[14] Y. Kutlu, and D. Kuntalp, "Feature extraction for ECG heartbeats using higher order statistics and WPD coefficients," Computer Methods and Programs in Biomedicine, vol. 105, no. 3, pp. 257-267, Mar. 2012.

[15] Z. Zhang, J. Dong, X. Luo, K. -S. Choi, and X. Wu, "Heartbeat classification using disease-specific feature selection," Computers in Biology and Medicine, vol. 46, pp. 79-89, Mar. 2014.

[16] N. Kishore, and S. Singh, "Cardiac analysis and classification of ECG signal using GA and NN," International Journal of Computer Applications, vol. 127, no. 12, pp. 23-27, Oct. 2015.

[17] A. Özbeyaz, and S. Arıca, "Familiar/unfamiliar face classification from EEG signals by utilizing pairwise distant channels and distinctive time interval”, SIViP, vol. 12, pp. 1181-1188, Mar. 2018.

[18] A. Özbeyaz, and S. Arıca, "Classification of EEG signals of familiar and unfamiliar face stimuli exploiting most discriminative channels", Turkish Journal of Engineering \& Computer Sciences, vol. 25, pp. 3342-3354, Jul. 2017.

[19] Q. Li, C. Rajagopalan, and G. D. Clifford, "A machine learning approach to multi-level ECG signal quality classification," Computer Methods and Programs in Biomedicine, vol. 117, no. 3, pp. 435-447, Dec. 2014.

[20] E. Passoli, and F. Melgani, "Genetic algorithm-based method for mitigating label noise issue in ECG signal classification," Biomedical Signal Processing and Control, vol. 19, pp. 130-136, May 2015.

[21] E. K. Roonizii, and R. Sassi, "A signal decomposition model-based bayesian framework for ECG components separation," IEEE Transactions on Signal Processing, vol. 64, no. 3, pp. 665-674, Feb. 2016.

[22] M. Thomas, M. K. Das, and S. Ari, "Automatic ECH arrhythmia classification using dual tree complex wavelet-based features," $A E U$ - International Journal of Electronics and Communications, vol. 69, no. 4, pp. 715-721, Apr. 2015.

[23] M. Alfaras, M. C. Soriano, and S. Ortlin, "A fast machine learning model for ECG-Based heartbeat classification and arrhythmia detection," Frontiers in Physics, vol. 7, pp. 1-11, Jul. 2019.

[24] A. Minchole, J. Camps, A. Lyon, and B. Rodriguez, "Machine learning in the electrocardiogram," Journal of Electrocardiogram, 
vol. 57, pp. 561-564, Nov.-Dec. 2019.

[25] F. I. Alarsan, and M. Younes, "Analysis and classification of heart diseases using heartbeat features and machine learning algorithms," Journal of Big Data, vol. 6, no. 81, pp. 1-15, Aug. 2019.

[26] R. M. Haralick, K. Shanmugam, and I. Dinstein, "Textural features for image classification," IEEE Transaction on Systems, Man and Cybernetics, vol. SMC-3, no. 6, pp. 610-621, Nov. 1973.

[27] G. B. Moody, and R. G. Mark, "The impact of the MIT-BIH Arrhythmia Database," IEEE Engineering in Medicine and Biology Magazine, vol. 20, no. 3, pp. 45-50, May-Jun. 2001.

[28] A. L. Goldberger, L. A. N. Amaral, L. Glass, J. M. Hausdorff, P. C. Ivanov, R. G. Mark, J. E. Mietus, G. B. Moody, C. K. Peng, H. E. Stanley, "PhysioBank, PhysioToolkit, and PhysioNet: Components of a New Research Resource for Complex Physiologic Signals," Circulation, vol. 101, no. 23, pp. 1-6, Jun. 2000.

[29] C. Antzelevitch, and A. Burashnikov, "Overview of basic mechanism of cardiac arrhythmia", Cardiac Electrophysiology Clinics, vol. 3, no. 1, pp. 23-45, Mar. 2011.

[30] M. Alfaouri, and K. Daqrouq, "ECG signal denoising by wavelet transform thresholding," American Journal of Applied Sciences, vol. 5, no. 3, pp. 276-281, Mar. 2008.

[31] S. W. Smith "The Scientist and Engineer's Guide to Digital Signal Processing," 2nd ed., California, USA: California Technical Publishing, 1999, pp. 277-282.

[32] J. O. Smith III, "Introduction to Digital Filters with Audio Applications," USA: W3K Publishing, http://books.w3k.org/, 2007. ISBN 978-0-9745607-1-7.

[33] A.Kalauzi, S. Spasic, M. Culic, G. Frbic, and L. J. Martac, "Consecutive differences as a method of signal fractal analysis", Fractals, vol. 13, no. 4, pp. 283-292, May. 2005.

[34] S. V. Stehman, "Selecting and interpreting measures of thematic classification accuracy," Remote Sensing of Environment, vol. 62, no. 1, pp. 77-89, Oct. 1997.

[35] T. Fawcett, "An introduction to ROC analysis," Pattern Recognition Letters, vol. 27, no. 8, pp. 861-874, Jun. 2006.

[36] M. Wasimuddin, K. Elleithy, A. S. Abuzneid, M. Faezipour, and O. Abuzaghleh, "Stages-Based ECG Signal Analysis From Traditional Signal Processing to Machine Learning Approaches: A Survey," IEEE Access, vol. 8, pp. 177782-177803, Sep. 2020.

[37] N. Fujita, A. Sato, and M. Kawarasaki, "Performance study of wavelet based ECG analysis for ST-segment detection," in Proc. 38th Int. Conf. Telecommun. Signal Process. (TSP), Prag, Czech Republic, 2015, pp. 430-434.

[38] L. Y. Di Marco, and L. Chiari, "A wavelet-based ECG delineation algorithm for 32-bit integer online processing," BioMedical Engineering OnLine, vol. 10, no. 23, pp. 1-19, Apr. 2011.

[39] J. P. Martinez, R. Almeida, S. Olmos, A. P. Rocha, and P. Laguna, "A wavelet-based ECG delineator: Evaluation on standard databases," IEEE Transactions on Biomedical Engineering, vol. 51, no. 4, pp. 570-581, Mar. 2004.

[40] Z. Zidelmal, A. Amirou, M. Adnane, and A. Belouchrani, "QRS detection based on wavelet coefficients," Computer Methods and Programs in Biomedicine, vol. 107, no. 3, pp. 490-496, Sep. 2012.

[41] A. Ghaffari, M. R. Homaeinezhad, and M. M. Daevaeiha, "High resolution ambulatory holter ECG events detection-delineation via modified multi-lead wavelet-based features analysis: Detection and quantification of heart rate turbulence," Expert Systems with Applications, vol. 38, no. 5, pp. 5299-5310, May 2011.

[42] B. Subramanian, "ECG signal classification and parameter estimation using multiwavelet transform," Biomedical Research, vol. 28, no. 7, pp. 3187-3193, 2017.

[43] F. Zhang, and Y. Lian, "QRS detection based on multiscale mathematical morphology for wearable ECG devices in body area networks," IEEE Transactions on Biomedical Circuits and Systems, vol. 3, no. 4, pp. 220-228, Jun. 2009.

[44] C. F. Zhang, and T. -W. Bae, "VLSI friendly ECG QRS complex detector for body sensor networks," IEEE Journal on Emerging and Selected Topics in Circuits and Systems, vol. 2, no. 1, pp. 52-59, Mar. 2012.

[45] A. E. Curtin, K. V. Burns, A. J. Bank, and T. I. Netoff, "QRS complex detection and measurement algorithms for multichannel ECGs in cardiac resynchronization therapy patients," IEEE Journal of Translational Engineering in Health and Medicine, vol. 6, pp. 111, Jun. 2018

[46] D. B. Saadi, G. Tanev, M. Flintrup, A. Osmanagic, K. Egstrup, K. Hoppe, P. Jennum, J. L. Jeppesen, H. K. Iversen, and H. B. D. Sorensen, "Automatic real-time embedded QRS complex detection for a novel patch-type electrocardiogram recorder," IEEE Journal of Translational Engineering in Health and Medicine, vol. 3, pp. 1-12, Apr. 2015.

[47] S. Panicker, and P. Gayathri, "Use of Machine Learning Techniques in Healthcare: A Brief Review of Cardiovascular Disease Classification," in 2nd International Conference on Communication and Information Processing (ICCIP-2020), 2020, pp. 1-12.

[48] C. K. Roopa, and B. S. Harish, "A survey on various machine learning approaches for ECG analysis," International Journal of Computer Applications, vol. 163, no. 9, pp. 25-33, Apr. 2017.

[49] P. Pławiak, "Novel genetic ensembles of classifiers applied to myocardium dysfunction recognition based on ECG signals,' Swarm and Evolutionary Computation, vol. 39, pp. 192-208, Apr. 2018

[50] W. Lu, H. Hou, J. Chu, "Feature fusion for imbalanced ECG data analysis," Biomedical Signal Processing and Control, vol. 41, pp. 152-160, Mar. 2018

[51] J. A. Nasiri, M. Naghibzadeh, H. S. Yazdi, and B. Naghibzadeh "ECG Arrhythmia Classification with Support Vector Machines and Genetic Algorithm". In Third UKSim European Symposium on Computer Modeling and Simulation, Athens, Greece, 2009, pp. 187192.

[52] S. Dalal, and R. Birok, "Analysis of ECG Signals using Hybric Classifier," International Advanced Research Journal in Science, Engineering and Technology, vol. 3, no. 7, pp. 89-95, Jul. 2006.

[53] V. Priyadharshini, and S. S. Kumar, "An Enhanced Approach on ECG Data Analysis using Improvised Genetic Algorithm," International Research Journal of Engineering and Technology (IRJET), vol. 2, no. 5, pp. 1248-1256, Aug. 2015. 